\title{
Perfil nutricional e socioeconômico de idosos admitidos no pronto atendimento médico de um hospital universitário
}

\section{Nutritional and socioeconomic profile of the elderly admitted in the medical care of a university hospital}

\section{Perfil nutricional y socioeconómico de ancianos admitidos en la pronta atención médica de un hospital universitario}

\author{
Danielle Rolim Ferreira Gomes ${ }^{1}$ \\ Fernanda Zanoni Cônsolo² \\ Fabiane La Flor Ziegler Sanches ${ }^{3}$
}

${ }^{1}$ Pós-graduanda em Nutrição Clínica pela Universidade Estácio de Sá. Especialização em Intensivismo e Urgência e Emergência pelo Programa de Residência Multiprofissional em Atenção ao Paciente Crítico do Hospital Universitário Maria Aparecida Pedrossian, Universidade Federal de Mato Grosso do Sul (HUMAP/UFMS). Graduada em Nutrição pela Universidade Anhanguera-

Uniderp. Docente do curso de Nutrição da Universidade Anhanguera-Uniderp. E-mail: danirolimfg@gmail.com, ORCID: http://orcid.org/0000-0001-6826-9530

${ }^{2}$ Doutora e mestre em Saúde e Desenvolvimento na Região Centro-Oeste pela Universidade Federal de Mato Grosso do Sul (UFMS). Pós-graduada em Nutrição Clínica. Graduada em Nutrição pela Universidade Católica

Dom Bosco. Professora adjunta do curso de Nutrição da UFMS. Tutora e docente do Programa de Residência Multiprofissional em Saúde, área

de concentração: Atenção ao Paciente Crítico (PREMUS-APC). E-mail: fernandazanoni@yahoo.com.br, ORCID: http://orcid.org/0000-0002-0812-2817

${ }^{3}$ Doutora e mestre em Alimentos e Nutrição pela Universidade Estadual de Campinas (UNICAMP). Especialista em Terapia Nutricional e Nutrição Clínica pelo Ganep Nutrição Humana. Graduada em Nutrição pelo Centro Universitário Franciscano (UNIFRA) e em Farmácia pela Universidade Federal de Santa Maria (UFSM). Professora adjunta III da

Faculdade de Ciências Farmacêuticas, Alimentos e Nutrição (FACFAN) da Universidade Federal de Mato Grosso do Sul (UFMS). Coordenadora de Gestão Acadêmica da FACFAN. Tem experiência na área de Nutrição Clínica, Nutrição Esportiva, Ciências de Alimentos e Experimental. E-mail: fabianelaflor@gmail.com, ORCID: http://orcid.org/0000-0002-0096-3697 
Resumo: A população mundial passa por processo de envelhecimento, sendo o idoso mais susceptível a alterações do estado nutricional, devendo ser sempre avaliado para que haja intervenção precoce. Objetivou-se traçar o perfil nutricional e socioeconômico de idosos admitidos no pronto atendimento médico de um hospital universitário. Tratase de estudo transversal, prospectivo, amostra por conveniência, realizado nas primeiras 48 horas de internação nas áreas verde, amarela e vermelha do Sistema Único de Saúde. Foram avaliados 100 idosos de ambos os sexos, por meio de formulário socioeconômico, triagem $\mathrm{MNA}^{\circledR}$-SF e avaliação nutricional. Observou-se uma maioria desnutrida ou sob risco nutricional, com prevalência masculina e forte relação com perda de peso, diminuição da ingesta, estresse ou doença aguda. Assim, constatou-se maior sensibilidade da MNA ${ }^{{ }_{-}}$ SF na identificação precoce do risco nutricional mesmo nos pacientes eutróficos ou em sobrepeso pelo IMC. Sugerem-se mais estudos associando transtornos neuropsicológicos em idosos e sua influência no estado nutricional na hospitalização.

Palavras-chave: hospitalização; estado nutricional; desnutrição.

Abstract: The world population goes through an aging process, with the elderly being more susceptible to changes in nutritional status; therefore, they should always be evaluated for early intervention. The objective was to trace the nutritional and socioeconomic profile of elderly people admitted to the emergency medical service of a university hospital. This is a cross-sectional, prospective study, sample by convenience, carried out in the first 48 hours of hospitalization in the green, yellow, and red areas of the Brazilian National Health Service. We evaluated 100 elderly people of both sexes, through a socioeconomic form, $\mathrm{MNA}^{\circledR}$-SF screening, and nutritional assessment. There was a malnourished majority or under nutritional risk, with a male prevalence and a strong relationship with weight loss, decreased intake, stress, and acute illness. Thus, there was greater sensitivity of MNA ${ }^{\circledR}$-SF in the early identification of nutritional risk even in eutrophic or overweight patients by BMI. It is suggested to undertake further studies associating neuropsychological disorders in the elderly and their influence on nutritional status at hospitalization.

Keywords: hospitalization; nutritional status; malnutrition.

Resumen: La población mundial pasa por un proceso de envejecimiento, los ancianos son más susceptibles a los cambios en el estado nutricional y siempre deben ser evaluados para una intervención temprana. Se objetivó rastrear el perfil nutricional y socioeconómico de las personas mayores ingresadas en el servicio médico de emergencia de un hospital universitario. Este es un estudio transversal, prospectivo, muestra por conveniencia, realizado en las primeras 48 horas de hospitalización en las áreas verde, amarilla y roja del Sistema Único de Salud. Se evaluaron 100 ancianos de ambos los sexos, a través de formulario socioeconómico, clasificación MNA ${ }^{\circledR}$-SF y evaluación nutricional. Se observó una mayoría desnutrida o en riesgo nutricional, con una prevalencia masculina y una fuerte relación con la pérdida de peso, disminución de la ingesta, estrés y enfermedades agudas. Por lo tanto, hubo una mayor sensibilidad de $\mathrm{MNA}^{\circledR}$-SF en la identificación temprana del riesgo nutricional incluso en pacientes eutróficos o con sobrepeso por el IMC. Se sugieren estudios adicionales que asocian trastornos neuropsicológicos en los ancianos y su influencia en el estado nutricional durante la hospitalización.

Palabras clave: hospitalización; estado nutricional; desnutrición. 


\section{INTRODUÇÃO}

A proporção de pessoas com 60 anos ou mais está crescendo rapidamente em todo o mundo, superando as outras faixas etárias. Em 2025, existirá no mundo aproximadamente 1,2 bilhão de pessoas com mais de 60 anos e, segundo o IBGE, em 2017, a população brasileira de idosos ultrapassou a marca de 30,2 milhões, sendo a maioria constituída por mulheres (56\%).

Em decorrência das mudanças compatíveis com o envelhecimento, o idoso torna-se mais suscetível a alterações do estado nutricional, sendo fatores preponderantes: predisposição genética, estado inflamatório e nutricional, inatividade física e comorbidades. As comorbidades podem ser agravadas pela desnutrição ou podem ser a causa do agravo nutricional. Além disso, os idosos apresentam simultaneamente várias doenças e fazem uso de múltiplos medicamentos, ficando o estado nutricional ainda mais comprometido.

A desnutrição em idosos pode ser causada por: acesso limitado aos alimentos; dificuldades socioeconômicas; pouco conhecimento sobre alimentação e nutrição, bem como consequentes escolhas alimentares incorretas; doenças; medicações; perda de dentição; isolamento social; deficiências físicas ou cognitivas que dificultem a capacidade de comprar e preparar suas refeições; falta de atividade física; e situações de emergência.

O Inquérito Brasileiro de Avaliação Nutricional Hospitalar (IBRANUTRI), um grande estudo realizado em 1996, com 4.000 pacientes hospitalizados, em que se avaliou o estado nutricional, revelou que a prevalência de desnutrição foi de $48,1 \%$, sendo $12,6 \%$ classificados com desnutrição grave. A amostra analisada era composta por $36 \%$ de idosos e foi associada a uma maior prevalência de desnutrição, quando comparada com aqueles com menos de 60 anos.

No intuito de detectar o risco nutricional ou mesmo a desnutrição precocemente e prevenir seus agravos, os indivíduos devem ser avaliados periodicamente, para que, em tempo hábil, sejam reconhecidos os distúrbios nutricionais e haja intervenção adequada. A triagem nutricional é um instrumento fundamental e sensível para detectar idosos que necessitem de avaliação nutricional completa e de uma terapia nutricional agressiva e 
imediata. Portanto, a identificação do risco nutricional no idoso pela triagem é indispensável para uma intervenção eficaz.

A European Society for Clinical Nutrition and Metabolism (ESPEN), recomenda, desde 2002, o uso da triagem Mini Avaliação Nutricional Reduzida (MNA ${ }^{\circledR}$-SF) para identificação do risco de desnutrição em idosos precocemente, uma vez que é uma ferramenta de triagem nutricional que inclui aspectos físicos e mentais os quais, frequentemente, afetam o estado nutricional do idoso. A MNA ${ }^{\circledR}$-SF serve como uma ferramenta válida e simples de rastrear de forma rápida os pacientes idosos em risco de desnutrição.

Países em desenvolvimento, como o Brasil, não se demonstram capacitados para o envelhecimento populacional, que exige preparação para as consequências econômicas e sociais que dele provêm. As altas taxas de desnutrição hospitalar em idosos podem ser atenuadas, se identificadas de maneira precoce, contribuindo para um menor tempo de internação hospitalar, complicações e mortalidade.

Diante do exposto, o objetivo do trabalho foi traçar o perfil nutricional e socioeconômico de idosos admitidos no Pronto Atendimento Médico (PAM) de um hospital universitário.

\section{METODOLOGIA}

Trata-se de um estudo transversal, prospectivo, de amostra por conveniência, realizado no período de abril a agosto de 2017, nas primeiras 48 horas de internação dos pacientes. A coleta foi realizada no Pronto Atendimento Médico de um hospital universitário, nas áreas de atendimento classificadas como verde, amarela e vermelha, conforme determina o Sistema Único de Saúde (SUS), na cidade de Campo Grande, Mato Grosso do Sul.

Participaram do estudo indivíduos de ambos os sexos, de idade igual ou superior a 60 anos, que aceitaram participar do estudo após a assinatura do Termo de Consentimento Livre e Esclarecido (TCLE). Foram excluídos da pesquisa indivíduos que apresentaram alteração do estado mental e que estavam sem o acompanhamento de um responsável para assinatura do TCLE. Este trabalho foi aprovado pelo Comitê de Ética em Pesquisa (CEP) da Universidade Federal de Mato Grosso do Sul, sob o protocolo de pesquisa número 1.902.648. 
A coleta de dados ocorreu por meio da aplicação de dois formulários. O primeiro, de autoria própria, continha informações gerais socioeconômicas de cada participante e também dados antropométricos, como peso, altura, altura do joelho, circunferência do braço (CB) e da panturrilha (CP), conforme Figura 1. Já no segundo a triagem nutricional dos idosos foi realizada por meio da Mini Avaliação Nutricional Reduzida (MNA ${ }^{\circledR}$-SF), ferramenta validada e mundialmente utilizada em estudos com indivíduos neste ciclo da vida, conforme Figura 2.

Figura 1 - Formulário de coleta de dados socioeconômicos dos participantes idosos avaliados $(n=100)$ no Pronto Atendimento Médico em Campo Grande, MS, Brasil, 2017

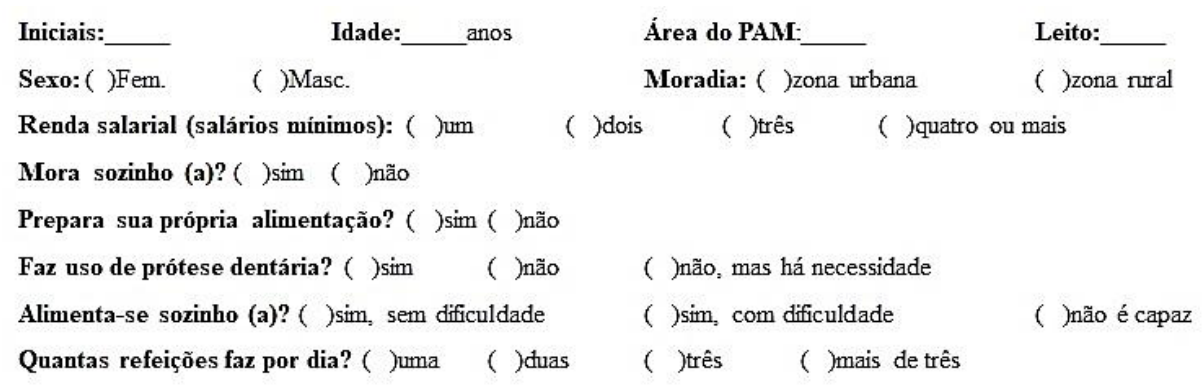

Utiliza três ou mais medicações diferentes prescritas por dia? ( ) sim $\quad($ )não

\section{DADOS ANTROPOMÉTRICOS}
Peso:
Altura:
CP: $\mathrm{cm}$
CB: $\_\mathrm{cm}$

Fonte: Autoria própria. 
Figura 2 - Mini Avaliação Nutricional Reduzida (MNA ${ }^{\circledR}$-SF) aplicada aos participantes idosos avaliados $(n=100)$ no Pronto Atendimento Médico em Campo Grande, MS, Brasil, 2017

\section{Mini Nutritional Assessment MNA $^{\circledR}$}

\section{Nestlé NutritionInstitute}

\begin{tabular}{|c|c|c|c|c|c|}
\hline Apelido: & & & Nome: & & \\
\hline Sexo: & Idade: & Peso, kg: & & Altura, cm: & Data: \\
\hline
\end{tabular}

Responda à secção "triagem", preenchendo as caixas com os números adequados. Some os números para obter a pontuação final da triagem.

\section{Triagem}

A Nos últimos três meses houve diminuição da ingesta alimentar devido a perda de apetite, problemas digestivos ou dificuldade para mastigar ou deglutir?

$0=$ diminuição grave da ingesta

1 = diminuição moderada da ingesta

2 = sem diminuição da ingesta

B Perda de peso nos últimos 3 meses

$0=$ superior a três quilos

1 = não sabe informar

$2=$ entre um e três quilos

$3=$ sem perda de peso

C Mobilidade

$0=$ restrito ao leito ou à cadeira de rodas

1 = deambula mas não é capaz de sair de casa

$2=$ normal

D Passou por algum stress psicológico ou doença aguda nos últimos três meses? $0=\operatorname{sim} \quad 2=$ não

E Problemas neuropsicológicos

0 = demência ou depressão graves

$1=$ demência ligeira

$2=$ sem problemas psicológicos

F1 Índice de Massa Corporal (IMC) $=$ peso em $\mathrm{kg} /($ estatura em m)

$0=$ IMC $<19$

$1=19 \leq I M C<21$

$2=21 \leq I M C<23$

$3=I M C \geq 23$.

SE IMC NÃO DISPONIVEL, SUBSTITUIR A QUESTÃO F1 PELA QUESTÃO F2.

NĀO RESPONDER À QUESTÃO F2 SE A QUESTÃO F1 JÁ ESTIVER COMPLETA

F2 Circunferência da Perna (CP) em cm

$0=\mathrm{CP}$ menor que 31

$3=\mathrm{CP}$ maior ou igual a 31

Pontuação da Triagem (subtotal, máximo de 14 pontos)

12-14 pontos: estado nutricional normal

8-11 pontos: sob risco de desnutrição

0-7 pontos: desnutrido

\section{References}

1. Vellas B, Villars H, Abellan G, et al. Overview of the MNA® - Its History and Challenges. J Nutr Health Aging. 2006;10:456-465

2. Rubenstein LZ, Harker JO, Salva A, Guigoz Y, Vellas B. Screening for Undernutrition in Geriatric Practice: Developing the Short-Form Mini

Nutritional Assessment (MNA-SF). J. Geront. 2001; 56A: M366-377

3. Guigoz $Y$. The Mini-Nutritional Assessment (MNA $)$ Review of the Literature - What does it tell us? J Nutr Health Aging. 2006; 10:466-487

4. Kaiser MJ, Bauer JM, Ramsch C, et al. Validation of the Mini Nutritional Assessment Short-Form (MNAB-SF): A practical tool for

identification of nutritional status. J Nutr Health Aging. 2009; 13:782-788.

(8) Société des Produits Nestlé SA, Trademark Owners.

๑ Société des Produits Nestlé SA 1994, Revision 2009.

Para maiores informaçð̄es: www.mna-elderly.com

Fonte: www.mna-elderly.com 
Perfil nutricional e socioeconômico de idosos admitidos no pronto atendimento médico de um hospital universitário

Ao final da triagem $\mathrm{MNA}^{\circledR}{ }^{\circledR} \mathrm{SF}$, o risco nutricional dos participantes foi determinado conforme consta no final da Figura 2.

A antropometria foi realizada no momento da aplicação do formulário e o peso foi aferido por meio de balança digital portátil Finlandek ${ }^{\circledR}$. Nos casos de edema, o excesso de peso hídrico foi estimado conforme recomendação de Duarte e Castelani (2002) e descontado do peso aferido.

A altura foi mensurada pela altura do joelho (AJ), medida com o paciente deitado em posição supina, com a perna esquerda flexionada formando um ângulo de $90^{\circ} \mathrm{com}$ o joelho. A mensuração ocorreu com a fita métrica inelástica posicionada entre a base do calcanhar e a parte superior da patela (MARUCCI; ALVES; GOMES, 2010). O valor encontrado foi utilizado na fórmula de Chumlea, Roche e Steinbaugh (1985) para encontrar o valor da estimativa de altura.

Os valores encontrados para peso e altura foram utilizados para cálculo do Índice de Massa Corporal (IMC), a partir da seguinte fórmula: IMC $\left(\mathrm{kg} / \mathrm{m}^{2}\right)=$ Peso $(\mathrm{kg}) /$ Estatura $\left(\mathrm{m}^{2}\right)$. Com base neste indicador, os idosos foram classificados segundo Lipschitz (1994): em magreza (IMC $<22 \mathrm{~kg}$ / $\mathrm{m}^{2}$ ), eutróficos (IMC de $22 \mathrm{~kg} / \mathrm{m}^{2}$ a $27 \mathrm{~kg} / \mathrm{m}^{2}$ ) e sobrepeso (IMC > $\left.27 \mathrm{~kg} / \mathrm{m}^{2}\right)$.

Conforme preconiza a $\mathrm{MNA}^{\circledR}$-SF, nos casos em que não foi possível determinar a aferição do IMC, foi realizada a circunferência da panturrilha (CP) com ponto de corte abaixo de $31 \mathrm{~cm}$ correspondendo à desnutrição. Caso o paciente apresentasse edema nas panturrilhas, a CP não era utilizada e o peso era estimado conforme as fórmulas de Ross Laboratories (2002), ou seja, utilizando altura do joelho e circunferência do braço. Não foi coletada a circunferência do braço (CB) de pacientes com edema nesta região.

Os dados coletados foram tratados pelo programa estatístico Statistical Package for the Social Sciences (SPSS), versão 20.0. A análise descritiva foi apresentada como média, desvio-padrão (DP) e/ou frequência absoluta e relativa. Os resultados foram analisados quanto a sua normalidade e posteriormente submetidos aos testes de Kolmogorov-Smirnov, Shapiro-Wilk, Mann-Whitney, Qui-Quadrado e teste exato de Fisher. Adotou-se como nível de significância $p<0,05$. 


\section{RESULTADOS E DISCUSSÃO}

Dos participantes selecionados, 14 foram excluídos, e participaram do estudo 100 indivíduos. As características gerais e socioeconômicas da população estudada estão descritas na Tabela 1.

Tabela 1 - Características gerais e socioeconômicas dos idosos avaliados ( $\mathrm{n}$ = 100) no Pronto Atendimento Médico em Campo Grande, MS, Brasil, 2017

\begin{tabular}{lcc}
\hline Dados avaliados & Unidades & Valor obtido \\
\hline Sexo & $\%(\mathrm{n})$ & \\
$\quad$ Feminino & $\%(\mathrm{n})$ & $53,0(47)$ \\
$\quad$ Masculino & $\mathrm{anos} \pm \mathrm{DP}$ & $71,04 \pm 8,57$ \\
Idade média & $\mathrm{kg}+\mathrm{DP}$ & $67,35 \pm 18,39$ \\
Peso corporal médio & $\mathrm{m}+\mathrm{DP}$ & $1,62 \pm 0,07$ \\
Altura média & $\mathrm{kg} / \mathrm{m}^{2}+\mathrm{DP}$ & $25,34 \pm 6,38$ \\
IMC médio & $\mathrm{cm}+\mathrm{DP}$ & $32,83 \pm 5,92$ \\
CP média & $\mathrm{cm}+\mathrm{DP}$ & $28,65 \pm 5,61$ \\
CB média & &
\end{tabular}

\section{Local de Internação}

PAM verde

$\begin{array}{lc}\%(n) & 76,0(76) \\ \%(n) & 22,0(22) \\ \%(n) & 2,0(2)\end{array}$

Tipo de moradia

Urbana

$\begin{array}{ll}\%(n) & 90,0(90) \\ \%(n) & 10,0(10)\end{array}$

\section{Renda Salarial}

1 salário

$\%(n)$

$61,0(61)$

2 salários

$\%(n)$

3 salários

$\%(n)$

$8,0(8)$

$\geq 4$ salários

$\%(n)$

$6,0(6)$

Mora sozinho?

Sim

$\%(n)$

$21,0(21)$

Não

$\%(n)$

$79,0(79)$

Prepara sua própria alimentação?

Sim

$\%(n)$

$57,0(57)$

Não

$\%(n)$

$43,0(43)$ 


\section{Dados avaliados \\ Faz uso de prótese dentária?}

Unidades

Valor obtido

Sim

$\%(n)$

$66,0(66)$

Não

$\%(n)$

$13,0(13)$

Não, mas há necessidade

$\%(n)$

21,0 (21)

Alimenta-se sozinho?

Sim, sem dificuldade

$\%(n)$

$94,0(94)$

Sim, com dificuldade

$\%(n)$

$6,0(6)$

Não é capaz

$\%(n)$

$0,0(0)$

N. de refeições/dia

1

$\%(n)$

4,0 (4)

2

$\%(n)$

$19,0(19)$

3

$\%(n)$

$37,0(37)$

$\geq 3$

$\%(n)$

40,0 (40)

Uso de $\geq 3$ medicações/dia

Sim

$\%(n)$

$67,0(67)$

Não

$\%(n)$

$33,0(33)$

\section{Classificação IMC}

Magreza

$\%(n)$

29,0 (29)

Eutrofia

$\%(n)$

$35,0(35)$

Sobrepeso

$\%(n)$

$36,0(36)$

Diminuição da ingesta nos últimos 3 meses

Severa

$\%(n)$

$40,0(40)$

Moderada

$\%(n)$

$16,0(16)$

Sem diminuição

$\%(n)$

44,0 (44)

Perda de peso nos últimos 3 meses

$>3 \mathrm{~kg}$

$\%(n)$

49,0 (49)

Não sabe informar

$\%(n)$

2,0 (2)

Entre 1 kg e 3 kg

$\%(n)$

$17,0(17)$

Sem perda

$\%(n)$

$32,0(32)$

\section{Mobilidade}

Restrito ao leito/cadeira de rodas

$\%(n)$

$6,0(6)$

Deambula, mas não sai de casa

$\%(n)$

24,0 (24)

Normal

$\%(n)$

$70,0(70)$

Estresse psicológico ou doença aguda nos últimos 3 meses

Sim 


\begin{tabular}{lcc}
\hline Dados avaliados & Unidades & Valor obtido \\
\hline Não & $\%(n)$ & $54,0(54)$ \\
Problemas neuropsicológicos & & \\
$\quad$ Demência/depressão graves & $\%(n)$ & $5,0(5)$ \\
$\quad$ Demência leve & $\%(n)$ & $12,0(12)$ \\
$\quad$ Sem problemas & $\%(n)$ & $83,0(83)$ \\
Resultado MNA ${ }^{\circledR}$-SF & & \\
$\quad$ Normal & $\%(n)$ & $31,0(31)$ \\
Sob risco de desnutrição & $\%(n)$ & $29,0(29)$ \\
$\quad$ Desnutrido & $\%(n)$ & $40,0(40)$ \\
\hline
\end{tabular}

Legenda: CP: circunferência da panturrilha; CB: circunferência do braço; IMC: Índice de Massa Corporal. n: número de participantes; \%: percentual de participantes; DP: desvio-padrão; kg: quilograma; $\mathbf{m}$ : metros; $\mathbf{k g} / \mathbf{m}^{\mathbf{2}}$ : quilograma por metro quadrado; $\mathbf{c m}$ : centímetros.Fonte: Autoria própria.

Não houve diferença significativa entre homens e mulheres para idade $(p=0,067)$, peso $(p=0,849)$ e CP $(p=0,834)$. Houve diferença estatística $(p<$ $0,05)$ entre os sexos em relação à altura $(p=0,0001)$, IMC $(p=0,005)$ e CB ( $p$ =0,024) (teste Mann-Whitney). Dos 100 indivíduos, foram coletados CB de 95 e CP de 61, a fim de estimar o peso corporal para dar o resultado da triagem.

Ao analisar a relação dos sexos com as variáveis nominais, observou-se que, do total de 100 pessoas, 47 eram do sexo feminino, das quais 33 $(70,2 \%)$ eram da área do PAM classificada como verde, $13(27,7 \%)$ da amarela e uma $(2,1 \%)$ da vermelha. Já o sexo masculino apresentou total de 53 , dos quais $43(81,1 \%)$ eram da área verde, nove $(17,0 \%)$ da amarela e um $(1,9 \%)$ da vermelha. Não houve diferença estatística entre os sexos e o local de internação no PAM ( $p=0,430$ pelo teste Qui-Quadrado).

Também não houve diferença estatística entre os sexos e o tipo de moradia - urbana ou rural $(p=0,640)$; entre os sexos e a renda salarial ( $p=$ $0,126)$; entre os sexos e o fato de morar sozinho $(p=0,578)$; entre os sexos e o uso de prótese dentária $(p=0,347)$; entre os sexos e o número de refeições/dia ( $p=0,564)$; entre os sexos e o uso de $\geq 3$ medicações diferentes/dia $(p=0,828)$; entre os sexos e a diminuição da ingesta alimentar $(p=0,352)$; entre os sexos e a perda de peso $(p=0,123)$; entre os sexos e a mobilidade $(p=0,080)$; entre os sexos e a existência de problemas neuropsicológicos 
Perfil nutricional e socioeconômico de idosos admitidos no pronto atendimento médico de um

$(p=0,340)$; entre os sexos e o resultado da MNA ${ }^{\circledR}$-SF $(p=0,550$ pelo teste Qui-Quadrado); e, por fim, entre os sexos e a capacidade de se alimentar sozinho ( $p=1,000$ pelo teste exato de Fisher).

Em contrapartida, houve associação significativa entre as variáveis nominais descritas na Tabela 2.

Tabela 2 - Comparação entre os sexos e as variáveis nominais com associações significativas dos idosos avaliados $(n=100)$ no Pronto Atendimento Médico em Campo Grande, MS, Brasil, 2017

\begin{tabular}{lccc}
\hline & $\begin{array}{c}\text { Mulheres } \\
(\mathbf{n}=\mathbf{4 7})\end{array}$ & $\begin{array}{c}\text { Homens } \\
(\mathbf{n}=\mathbf{5 3})\end{array}$ & $\begin{array}{c}\mathbf{p}^{*} \\
\mathbf{1 < 0 , 0 5}\end{array}$ \\
\hline $\begin{array}{l}\text { Prepara sua própria alimentação? } \\
\text { Sim \% (n) }\end{array}$ & $83,0(39)$ & $34,0(18)$ & 0,0001 \\
$\quad$ Não \% (n) & $17,0(8)$ & $66,0(35)$ & \\
Classificação do IMC & & & \\
$\quad$ Magreza \% (n) & $23,4(11)$ & $34,0(18)$ & \\
$\quad$ Eutrofia \% (n) & $27,7(13)$ & $41,5(22)$ & 0,040 \\
$\quad$ Sobrepeso \% (n) & $48,9(23)$ & $24,5(13)$ & \\
Estresse psicológico/doença aguda & & & \\
nos últimos 3 meses & & & \\
$\quad$ Sim \% (n) & $59,6(28)$ & $34,0(18)$ & 0,010 \\
$\quad$ Não \% (n) & $40,4(19)$ & $66,0(35)$ & \\
\hline
\end{tabular}

Legenda: $\mathbf{n : ~ n u ́ m e r o ~ d e ~ p a r t i c i p a n t e s ; ~ \% : ~ p e r c e n t u a l ~ d e ~ p a r t i c i p a n t e s ; ~ * T e s t e ~ Q u i - Q u a d r a d o . ~}$ Fonte: Autoria própria.

Ao comparar o resultado da $\mathrm{MNA}^{\circledR}$-SF com as outras variáveis nominais, não houve diferença estatística pelo teste Qui-Quadrado, ou seja, entre os resultados deste estudo com: os diferentes sexos ( $p=0,550)$; as áreas de internação do PAM ( $p=0,640)$; o tipo de moradia $(p=0,262)$; o valor da renda $(p=0,923)$; o fato de morar sozinho $(p=0,542)$; o fato de saber cozinhar $(p=0,277)$; o uso de prótese dentária $(p=0,462)$; a capacidade de se alimentar sozinho ( $p=0,388)$; o número de refeições realizadas/dia $(p=0,386)$; e a utilização de mais de três medicamentos/dia $(p=0,068)$.

Em compensação, houve diferença significativa entre as variáveis descritas na Tabela 3. 
Tabela 3 - Comparação entre o resultado da $\mathrm{MNA}^{\circledR}$-SF e as variáveis socioeconômicas, clínicas e nutricionais dos idosos avaliados $(n=100)$ no Pronto Atendimento Médico em Campo Grande, MS, Brasil, 2017

\begin{tabular}{|c|c|c|c|c|}
\hline \multicolumn{5}{|c|}{ Resultado $\mathrm{MNA}^{\circledR}-\mathrm{SF}$} \\
\hline & $\begin{array}{l}\text { Normal } \\
(n=31)\end{array}$ & $\begin{array}{c}\text { Risco de } \\
\text { desnutrição } \\
(n=29)\end{array}$ & $\begin{array}{l}\text { Desnutrido } \\
(n=40)\end{array}$ & $\begin{array}{c}p^{*} \\
(<0,05)\end{array}$ \\
\hline \multicolumn{5}{|l|}{$\begin{array}{l}\text { Perda de peso nos } \\
\text { últimos } 3 \text { meses }\end{array}$} \\
\hline$>3 \mathrm{~kg} \%(\mathrm{n})$ & $0,0(0)$ & $41,4(12)$ & $92,5(37)$ & \multirow[b]{2}{*}{0,0001} \\
\hline $\begin{array}{l}\text { Não sabe informar \% (n) } \\
\text { Entre } 1 \mathrm{~kg} \text { e } 3 \mathrm{~kg} \%(\mathrm{n}) \\
\text { Sem perdas \% (n) }\end{array}$ & $\begin{array}{c}3,2(1) \\
19,4(6) \\
77,4(24)\end{array}$ & $\begin{array}{l}3,4(1) \\
31,0(9) \\
24,1(7)\end{array}$ & $\begin{array}{l}0,0(0) \\
5,0(2) \\
2,5(1)\end{array}$ & \\
\hline \multicolumn{5}{|l|}{ Classificação do IMC } \\
\hline Magreza \% (n) & $3,2(1)$ & $20,7(6)$ & $55,0(22)$ & \multirow{3}{*}{0,0001} \\
\hline Eutrofia \% (n) & $48,4(15)$ & $31,0(9)$ & $27,5(11)$ & \\
\hline Sobrepeso \% (n) & $48,4(15)$ & $48,3(14)$ & $17,5(7)$ & \\
\hline \multicolumn{5}{|l|}{$\begin{array}{l}\text { Diminuição da ingestão } \\
\text { alimentar nos últimos } 3 \\
\text { meses }\end{array}$} \\
\hline Severa \% (n) & $3,2(1)$ & $34,5(10)$ & $72,5(29)$ & \multirow{3}{*}{0,0001} \\
\hline Moderada \% (n) & $12,9(4)$ & $24,1(7)$ & $12,5(5)$ & \\
\hline Sem diminuição \% (n) & $83,9(26)$ & $41,4(12)$ & $15,0(6)$ & \\
\hline \multicolumn{5}{|l|}{$\begin{array}{l}\text { Estresse psicológico/ } \\
\text { doença aguda nos últi- } \\
\text { mos } 3 \text { meses }\end{array}$} \\
\hline $\operatorname{Sim} \%(n)$ & $32,3(10)$ & $37,9(11)$ & $62,5(25)$ & \multirow{3}{*}{0,024} \\
\hline Não \% (n) & $67,7(21)$ & $62,1(18)$ & $37,5(15)$ & \\
\hline \multicolumn{4}{|l|}{$\begin{array}{l}\text { Problemas } \\
\text { neuropsicológicos }\end{array}$} & \\
\hline $\begin{array}{l}\text { Demência/depressão } \\
\text { graves \% (n) }\end{array}$ & $0,0(0)$ & $3,4(1)$ & $10,0(4)$ & \multirow{3}{*}{0,0001} \\
\hline Demência leve \% (n) & $0,0(0)$ & $3,4(1)$ & $27,5(11)$ & \\
\hline Sem problemas \% (n) & $100(31)$ & $93,1(27)$ & $62,5(25)$ & \\
\hline
\end{tabular}

Legenda: $\mathbf{n}$ : número de participantes; \%: percentual de participantes; *Teste Qui-Quadrado. Fonte: Autoria própria.

Em relação à classificação do estado nutricional pela $\mathrm{MNA}^{{ }^{\circledR}}$-SF, observou-se neste estudo uma maioria desnutrida $(40,0 \%)$ e em risco de 
Perfil nutricional e socioeconômico de idosos admitidos no pronto atendimento médico de um hospital universitário

desnutrição (29,0\%) em relação aos eutróficos (31\%). Quanto ao estado nutricional das mulheres, 17 (36,2\%) estavam normais, 12 (25,5\%) sob risco de desnutrição e $18(38,3 \%)$ desnutridas. Já entre os homens ( $n=53), 14(26,4 \%)$ apresentavam estado nutricional normal, $17(32,1 \%)$ sob risco de desnutrição e 22 (41,5\%) desnutridos. Os estudiosos Pereira, Spyrides e Andrade (2016) também observaram relação entre baixo peso e o sexo masculino.

Os resultados do presente estudo se compararam aos resultados do grande estudo brasileiro BRAINS, realizado em 2013, em que, dos 19.222 pacientes avaliados em 110 hospitais brasileiros, por meio de triagem nutricional, 10.234 foram submetidos à $\mathrm{MNA}^{\circledR}{ }^{\circledR}$ SF. Revelou-se que a maioria deles $(69,2 \%)$ foi classificada com risco de desnutrição $(38,4 \%)$ ou como desnutrida (30,8\%), e 30,8\% dos pacientes foram considerados eutróficos (BORGHI et al., 2013).

Um estudo realizado na Itália, com idosos institucionalizados, comparou a eficácia das triagens MUST, NRS 2002, MNA ${ }^{\circledR}$ completa e MNA ${ }^{\circledR}-S F$. Ao total, participaram 246 idosos (164 mulheres e 82 homens), sendo a maioria classificada em risco nutricional ou desnutrição pela $\mathrm{MNA}^{\circledR}{ }^{-S F}$ - resultado semelhante ao do presente estudo, porém, com prevalência de desnutrição no sexo feminino. Os autores chegaram à conclusão de que, ao comparar a MNA ${ }^{\circledR}$ completa com a $\mathrm{MNA}^{\circledR}$-SF, a última apresentou alta sensibilidade $(96,4 \%)$ e eficiência tanto quanto a completa, porém em menor tempo e maior facilidade de aplicação. A MNA ${ }^{\circledR}$-SF foi considerada como ferramenta de referência, e parâmetros psicológicos, funcionais e cognitivos são, provavelmente, os fatores de risco mais importantes para a desnutrição, em comparação com a doença aguda, em internações de longa duração (DONINI et al., 2016).

Outro estudo, realizado em Israel, avaliou 215 pacientes idosos internados em unidade ortogeriátrica, por meio da MNA ${ }^{\circledR}-S F$, MUST e NRS 2002. A maioria era feminina e, de acordo com a $M N A^{\circledR}-S F, 44,2 \%$ do total estavam sob risco nutricional e $11,6 \%$ estavam desnutridos. Em um período de 6 meses, 100 desses pacientes (46,5\%) foram readmitidos no hospital. A maioria deles $(64,0 \%)$ havia sido classificada como desnutrida ou sob risco de desnutrição pela $\mathrm{MNA}^{\circledR}$-SF, e 36,0\% foram considerados eutróficos ( $\mathrm{p}=$ $0,024)$. Durante 36 meses, a taxa de mortalidade foi de 36,7\% ( $n=79)$ e, segundo o resultado da $\mathrm{MNA}^{\circledR}{ }^{-} \mathrm{SF}$, a taxa de mortalidade foi menor no grupo 
dos eutróficos (22,1\%) em comparação aos desnutridos (50,5\%, p=0,0001) e com risco de desnutrição $(40 \%, p=0,01)$. Os pesquisadores chegaram à conclusão de que todas as ferramentas de triagem foram adequadas para identificar desnutrição em idosos operados com fratura de quadril, porém, somente a $\mathrm{MNA}^{\circledR}$-SF pôde prever as readmissões e a mortalidade, sendo, então, a ferramenta mais indicada (KOREN-HAKIM et al., 2016).

Resultado semelhante foi encontrado por Alzahrani e Alamri (2017) em estudo realizado em um hospital na Arábia Saudita, com 278 idosos hospitalizados, de prevalência feminina (60,5\%). Assim como no presente estudo, os pesquisadores supracitados, por meio da MNA ${ }^{\circledR}$-SF, observaram maioria de idosos desnutridos ou em risco de desnutrição $(76,6 \%)$ e analisaram que aqueles desnutridos tinham significativamente mais chances de serem reinternados, quando comparados aos eutróficos $(p=0,001)$. Além disso, das sete mortes registradas, cinco foram de idosos desnutridos.

Estudo realizado pela ASPEN, em 34 hospitais da Grécia, aplicou em 2.970 pacientes adultos e idosos as triagens NRS-2002, MUST e MNA ${ }^{\circledR}$-SF. Destes, 1.391 indivíduos foram triados pela MNA ${ }^{\circledR}$-SF $(54,1 \%$ do sexo masculino) e, entre as triagens, a $\mathrm{MNA}^{\circledR}{ }^{\circledR}$-SF foi a que detectou maior número de risco nutricional (60,5\%). O estudo observou que os idosos mais longevos (> 80 anos) foram os que mais apresentavam risco nutricional (DOUNDOULAKIS et al., 2017). O presente estudo não avaliou a população adulta, porém, apontou também que a maioria dos idosos já está em risco nutricional ou desnutrição no momento da admissão hospitalar, corroborando a importância da detecção precoce da desnutrição.

No que se refere ao resultado da $\mathrm{MNA}^{\circledR}{ }^{\circledR}$-SF e à forte associação significativa com a perda de peso nos últimos três meses ( $p=0,0001)$, este estudo se compara ao estudo de Koren-Hakim et al. (2016), que encontraram também associação significativa $(p=0,001)$ de acordo com os três tipos de triagens utilizadas. Esta associação foi encontrada, ainda, pelos pesquisadores Alzahrani e Alamri (2017) com relevância estatística ( $p=0,001)$. Atualmente, estudiosos que avaliaram também idosos hospitalizados ( $n=$ 235) por meio da $\mathrm{MNA}^{\circledR}$-SF corroboraram que a perda de peso e o declínio na mobilidade e na ingesta alimentar são os principais fatores de risco para desnutrição (RASHID; TIWARI; LEHL, 2020). 
Perfil nutricional e socioeconômico de idosos admitidos no pronto atendimento médico de um hospital universitário

No tocante ao resultado da $\mathrm{MNA}^{\circledR}$-SF e à classificação do IMC, notou-se associação significativa neste estudo $(p=0,0001)$. Um dado interessante foi que, entre os indivíduos eutróficos pelo IMC, 31,0\% foram classificados com risco nutricional pela triagem e $27,5 \%$ com desnutrição. E, dos pacientes em sobrepeso pelo IMC, 48,3\% foram diagnosticados pela $\mathrm{MNA}^{\circledR}$-SF com risco de desnutrição e 15\% com desnutrição. Resultados semelhantes foram observados por Koren-Hakim et al. (2016), Alzahrani e Alamri (2017) e por Ferreira, Guimarães e Sanches (2018). Esses dados apontam que a MNA ${ }^{\circledR}$-SF é capaz de detectar o risco nutricional e a desnutrição de forma precoce, pois leva em consideração fatores biopsicossociais que influenciam no estado nutricional (DONINI et al., 2016).

No que concerne à diminuição da ingestão alimentar nos últimos três meses e ao resultado da $\mathrm{MNA}^{\circledR}$-SF, observou-se, neste estudo, associação significativa ( $p=0,0001$ ), em que pacientes com diminuição severa da ingestão alimentar eram mais desnutridos. Resultado similar foi encontrado por Azevedo et al. (2007) e Koren-Hakim et al. (2016). Ao contrário do que se espera, Alzahrani e Alamri (2017), em seu estudo, observaram que, dos idosos desnutridos, apenas 1,2\% apresentou diminuição severa da ingesta, $54,2 \%$ tiveram diminuição moderada e 44,1\% não apresentaram diminuição.

Em relação à presença de estresse psicológico e doença aguda nos últimos três meses, no presente estudo foi encontrada associação significativa com a desnutrição ( $p=0,024)$, assim como Azevedo et al. (2007) encontraram também $(p=0,001)$. Poucos estudos fizeram a comparação entre as variáveis comentadas.

No que diz respeito às variáveis de problemas neuropsicológicos e ao resultado da $\mathrm{MNA}^{\circledR}{ }^{\circledR}$-SF, observou-se, neste estudo, diferentemente do que se espera em comparação com outros trabalhos da literatura, que, entre os indivíduos desnutridos, 62,5\% não tinham problemas neuropsicológicos, e dos sob risco de desnutrição, 93,1\% também não apresentaram. Evidências contrárias foram encontradas por Saka et al. (2010), que avaliaram idosos atendidos em ambulatório e observaram associação significativa entre desnutrição, demência ( $p=0,0001$ ) e depressão ( $p=0,0001)$, dentre outras síndromes geriátricas. Azevedo et al. (2007) identificaram também associação entre desnutrição e problemas neuropsicológicos $(p=0,032)$ 
em idosos hospitalizados. Os estudiosos Dent et al. (2017) fizeram associação significativa entre desnutrição e depressão $(p=0,008)$ em idosos hospitalizados, devido ao grande impacto no apetite, e apontam que poucos estudos investigaram essa associação em ambiente hospitalar. Um recente estudo espanhol multicêntrico, utilizando a $\mathrm{MNA}^{\circledR}$-SF em uma amostra de 749 idosos, encontrou também associação com risco nutricional e demência em idosos de um departamento de emergência cardíaca (MARTín SÁNCHEZ et al., 2019)

No presente estudo, não foi encontrada associação significativa entre desnutrição e o fato de morar sozinho, ter baixa renda ou tipo de moradia (zona urbana ou rural). Comparando os achados com a literatura, ao contrário do que se espera, Alzahrani e Alamri (2017) apontaram que 92,4\% dos idosos desnutridos e $59,7 \%$ dos sob risco de desnutrição moravam com mais pessoas $(p=0,001)$. A relação entre desnutrição, baixa renda e tipo de moradia também não foi encontrada por Azevedo et al. (2007). Por outro lado, um grande estudo realizado por Pereira, Spyrides e Andrade (2016), com 20.114 idosos participantes da Pesquisa de Orçamentos Familiares (POF) 2008/2009, do IBGE, encontrou relação diretamente proporcional entre baixo peso e moradia em zona rural e entre o fato de o idoso morar sozinho. Além disso, apontou associação positiva entre renda e IMC (quanto maior a renda, maior o IMC). Idosos que moram sozinhos apresentam tendência a problemas de saúde que podem interferir no apetite e na aquisição de alimentos, levando à desnutrição. Por outro lado, esses problemas podem induzir também ao sobrepeso se o consumo de alimentos for de baixa qualidade nutricional (FARES et al., 2012; PEREIRA; SPYRIDE; ANDRADE, 2016).

Poucos estudos relacionaram desnutrição e perda de mobilidade. No presente estudo, não foi encontrada associação significativa entre as variáveis. Porém, Azevedo et al. (2007) observaram associação significativa ( $p=0,001$ ) e Martín Sanchéz et al. (2019) apontaram que o risco nutricional era mais prevalente em idosos com dependência funcional grave.

O perfil epidemiológico e de mortalidade dos idosos está diretamente relacionado ao estado nutricional deste segmento populacional. Dada a alta prevalência de desnutrição e a falta de manejo adequado de pacientes idosos em muitos locais, a implementação de triagem nutricional de forma rotineira 
Perfil nutricional e socioeconômico de idosos admitidos no pronto atendimento médico de um hospital universitário

deve identificar de maneira precoce o risco nutricional desses pacientes, possibilitando uma estratégia de suporte nutricional. Recomenda-se que a triagem nutricional seja aplicada nas primeiras 48 horas de internação hospitalar. Além disso, deve haver ações de monitoramento e promoção de qualidade de vida e saúde a esta população (GYONNET; ROLLAND, 2015; PEREIRA; SPYRIDE; ANDRADE, 2016, CASTRO et al., 2018).

\section{CONSIDERAÇÕES FINAIS}

Conclui-se que o perfil nutricional dos idosos participantes deste estudo, internados em um hospital público de Mato Grosso do Sul, caracteriza-se por uma maioria masculina desnutrida ou sob risco de desnutrição, com perfil socioeconômico de baixa renda e que reside em moradia urbana. Constatou-se sofrerem influência em seu estado nutricional devido às diminuições em sua ingestão alimentar, perda de peso significativa antes da internação hospitalar e por estresse/doenças agudas. Adicionalmente, a triagem $\mathrm{MNA}^{\circledR}{ }^{\circledR}$-SF foi sensível em detectar risco nutricional ou desnutrição mesmo em pacientes classificados como eutróficos ou sobrepeso/obesidade, segundo o IMC.

Sabe-se que no Brasil, pela legislação vigente, é obrigatória a realização de triagem nutricional, embora muitos hospitais do país não utilizem essa ferramenta. Nesse sentido, o presente estudo corrobora a importância da identificação precoce daqueles que necessitam de intervenção nutricional imediata, e seus resultados podem ser utilizados pela instituição e por autoridades locais no âmbito da saúde pública, para planejar os serviços de saúde e implantar o uso de triagem nutricional não só para pacientes idosos, mas para todos, conforme exige a legislação, economizando, consequentemente, recursos financeiros em decorrência de menor tempo de hospitalização.

No que tange às limitações do estudo, houve dificuldade em classificar os idosos com problemas neuropsicológicos, visto as variadas formas de demência, e em encontrar literatura pertinente e atualizada para a discussão. Assim, sugere-se que mais estudos sejam realizados no tocante à associação de transtornos neuropsicológicos em idosos e sua influência no estado nutricional durante o período de hospitalização. 


\section{REFERÊNCIAS}

ALZAHRANI, S. H.; ALAMRI, S. H. Prevalence of malnutrition and associated factors among hospitalized elderly patients in King Abdulaziz University Hospital, Jeddah, Saudi Arabia. BMC Geriatrics, Arábia Saudita, v. 17, n. 136, p. 7, jul. 2017. Disponível em: https://bmcgeriatr.biomedcentral.com/track/ pdf/10.1186/s12877-017-0527-z. Acesso em: 18 jul. 2019.

AZEVEDO, L. C.; FENILLI, M.; NEVES, L. ALMEIDA, C. B.; FARIAS, M. B.; BREITKOPF, T.; SILVA, A. A.; ESMERALDINO, R. Principais fatores da mini-avaliação nutricional associada a alterações nutricionais de idosos hospitalizados. Arquivos Catarinenses de Medicina, Santa Catarina, v. 36, n. 3, p. 7-14, 2007. Disponível em: http://www.acm.org.br/acm/revista/pdf/ artigos/498.pdf. Acesso em: 28 ago. 2019.

BORGHI, R.; MEALE, M. M. S.; GOUVEIA, M. A. P.; FRANÇA, J. I. D.; DAMIÃO, A. O. M. C. Perfil nutricional de pacientes internados no Brasil: análise de 19.222 pacientes (Estudo BRAINS). Revista Brasileira de Nutrição Clínica, São Paulo, v. 28, n. 4, p. 255-63, out. 2013. Disponível em: http://www. braspen.com.br/home/wp-content/uploads/2016/12/01-Estudo-Teoricoda-composi\%C3\%A7\%C3\%A3o.pdf. Acesso em: 28 ago. 2019.

CASTRO, M. G.; RIBEIRO, P. C.; SOUZA, I. A. O.; CUNHA, H. F. R.; SILVA, M. H. N.; ROCHA, E. E. M.; CORREIA, F. G.; LOSS, S. H.; FRANCO FILHO, J. W.; NUNES, D. S. L.; GONÇALVES, R. C.; NUNES, L.; CENICCOLA, G. D.; TOLEDO, D. O. Diretriz Brasileira de Terapia Nutricional no Paciente Grave. Brazilian Society of Parenteral and Enteral Nutrition. BRASPEN Journal, São Paulo, v. 33, supl. 1, p. 2-36, jan./mar. 2018. Disponível em: https://www.braspen. org/braspen-journal-copia. Acesso em: 28 ago. 2019.

CHUMLEA, W. C.; ROCHE, A. F.; STEIBAUGH, M. L. Estimating stature from knee height for persons 60 to 90 years of age. Journal of the American Geriatrics Society, Ohio, v. 33, n. 2, p. 116-20, fev. 1985. Disponível em: https://doi. org/10.1111/j.1532-5415.1985.tb02276.x. Acesso em: 28 ago. 2019.

DENT, E.; WRIGHT, O.; HOOGENDIJK, E. O.; HUBBARD, R. E. Nutritional screening and dietitian consultation rates in a geriatric evaluation and management unit. Nutrition \& Dietetics, Australia, v. 75, n. 1, p. 1-6, nov. 2017. Disponível em: https://doi.org/10.1111/1747-0080.12391. Acesso em: 28 ago. 2019.

DONINI, L. M.; POGGIOGALLE, E.; MOLFINO, A.; ROSANO, A.; LENZI, A.; FANELLI, F. R.; MUSCARITOLI, M. Mini-nutritional assessment, malnutrition 
Perfil nutricional e socioeconômico de idosos admitidos no pronto atendimento médico de um

universal screening tool, and nutrition risk screening tool for the nutritional evaluation of older nursing home residents. Journal of the American Medical Directors Association (JAMDA), Italy, v. 17, p. 959.e11-959.e18, out. 2016. Disponível em: https://doi.org/10.1016/j.jamda.2016.06.028. Acesso em: 28 ago. 2019.

DOUNDOULAKIS, I.; POULIA, K. A.; ANTZA, C.; BOURAS, E.; KASAPIDOU, E.; KLEK, S.; CHOURDAKIS, M. Screening for Malnutrition among People Accessing Health Services at Greek Public Hospitals: Results from an Observational Multicenter Study. Journal of Parenteral and Enteral Nutrition, Greece, v. 20, n. 10, p. 1-9, jun./2017. Disponível em: https://doi. org/10.1177/0148607117722748. Acesso em: 28 ago. 2019.

DUARTE, A. C.; CASTELLANI, F. R. Semiologia Nutricional. 1. ed. Rio de Janeiro: Axcel Books do Brasil Ltda, 2002.

FARES, D.; BARBOSA, A. R.; BORGATTO, A. F.; COQUEIRO, R. S.; FERNANDES, M. H. Fatores associados ao estado nutricional de idosos de duas regiões do Brasil. Revista da Associação Médica Brasileira, Florianópolis, v. 58, n. 4, p. 434-41, abr. 2012. Disponível em: http://dx.doi.org/10.1590/S010442302012000400013. Acesso em: 28 ago. 2019.

FERREIRA, T.; GUIMARÃES, R. C. A.; SANCHES, F. L. F. Z. Risco nutricional em pacientes hospitalizados: comparação de três protocolos de triagem nutricional. Multitemas, Campo Grande, MS, v. 23, n. 55, p. 245-63, set./dez. 2018. Disponível em: http://www.multitemas.ucdb.br/article/ view/1606/1597. Acesso em: 28 ago. 2019.

GYONNET, S.; ROLLAND, Y. Screening for Malnutrition in Older People. Clinics in Geriatric Medicine, France, v. 31, n. 3, p. 429-37, ago. 2015. Disponível em: https://doi.org/10.1016/j.cger.2015.04.009. Acesso em: 28 ago. 2019.

KOREN-HAKIM, T.; WEISS, A.; HERSHKOVITZ, A.; OTZRATENI, I.; ANBAR, R.; NEVO, R. F. G.; SCHLESINGER, A.; FRISHMAN, S.; SALAI, M.; BELOOSESKY, Y. Comparing the adequacy of the MNA-SF, NRS-2002 and MUST nutritional tools in assessing malnutrition in hip fracture operated elderly patients. Clinical Nutrition, Israel, v. 35, p. 1053-58, out. 2016. Disponível em: https:// doi.org/10.1016/j.clnu.2015.07.014. Acesso em: 28 ago. 2019.

LIPSCHITZ, D. A. Screening for nutritional status in the elderly. Primary Care, Philadelphia, Saunders, v. 21, n. 1, p. 55-67, mar. 1994. 
MARUCCI, M. F. N.; ALVES, R. P.; GOMES, M. M. B. C. Nutrição em Gerontologia. In: SILVA, S. M. C. S.; MURA, J. D. P. cap. 25. Tratado de Alimentação, Nutrição \& Dietoterapia. 2. ed. São Paulo: Roca, 2010. p. 461-88.

MARTÍN-SÁNCHEZ, F. J.; TRIANA, F. C.; ROSSELLO, X.; GARCÍA, R. P.; GARCÍA, G. L.; CAIMARI, F.; VIDÁN, M. T.; ARTACHO, P. R.; CASTILLO, J. G.; LLORENS, P.; HERRERO, P.; JACOB, J.; GILA, V.; PÉREZ, C. F.; GIL, P.; BUENO, H.; MIRÓ, O.; MARTÍN, P. M.; ADRADA, E. R.; SANTOS, M. C.; SALGADO, L.; BRIZZI, B. N.; DOCAVO, M. L.; SUÁREZ-CADENAS, M. M.; XIPELL, C.; SÁNCHEZ, C.; AGUILÓ, S.; GAYTAN, J. M.; JEREZ, A.; PÉREZ-DURÁ, M. J.; GIL, P. B.; LÓPEZ-GRIMA, M. L.; VALERO, M. L.; VALERO, A.; AGUIRRE, A.; PEDRAGOSA, M. A.; PIÑERA, P.; AREGUES, P. L.; NICOLÁS, J. A. S.; RIZZI, M. A.; MATEO, S. H.; ALQUEZAR, A.; ROSET, A.; FERRER, C.; LLOPIS, F.; PÉREZ, J. M. A.; DIEZ, M. P. L.; RICHARD, F.; FERNÁNDEZ-CAÑADAS, J. M.; CARRATALÁ, J. M.; JAVALOYES, P.; ANDUEZA, J. A.; FERNÁNDEZ, J. A. S.; ROMERO, R.; LORANCA, M. M.; RODRÍGUEZ, V. A.; LORCA, M.T.; CALDERÓN, L.; FERRER, E. S.; GARRIDO, J. M.; MOJARRO, E. M. Effect of risk of malnutrition on 30-day mortality among older patients with acute heart failure in Emergency Departments. European Journal of Internal Medicine, Madrid, Spain, v. 65, p. 69-77, maio 2019. Disponível em: https:// doi.org/10.1016/j.ejim.2019.04.014. Acesso em: 17 jul. 2019.

PEREIRA, I. F. da S.; SPYRIDES, M. H. C.; ANDRADE, L. de M. B. Estado nutricional de idosos no Brasil: uma abordagem multinível. Cadernos de Saúde Pública, Natal, RN, v. 32, n. 5, p. 1-12, maio 2016. doi: http://dx.doi. org/10.1590/0102-311X00178814

RASHID, I.; TIWARI, P.; LEHL, S. S. Malnutrition among elderly a multifactorial condition to flourish: evidence from a cross-sectional study. Clinical Epidemiology and Global Health, India, v. 8, n. 1, p. 91-5, mar. 2020. Disponível em: https://doi.org/10.1016/j.cegh.2019.05.001. Acesso em: 5 ago. 2020.

ROSS LABORATORIES. The Ross Knee Height Caliper. Ross Products Division, Abbott Laboratories, Columbus, Ohio, fev. 2002. Disponível em: http:// priorart.ip.com/IPCOM/000006911/. Acesso em: 18 jul. 2019.

SAKA, B.; KAYA, O.; OZTURK, G. B.; ERTEN, N.; KARAN, M. A. Malnutrition in the elderly and its relationship with other geriatric syndromes. Clinical Nutrition, Istanbul, Turkey, v. 29, n. 6, p. 745-8, dez. 2010. Disponível em: https://doi.org/10.1016/j.clnu.2010.04.006. Acesso em: 28 ago. 2019. 